Maurice Kauffer

Université de Lorraine-Nancy/ATILF-CNRS

23 Boulevard Albert $1^{\mathrm{er}}$ BP 60446

54001 Nancy-Cedex, France

maurice.kauffer@univ-lorraine.fr

http://orcid.org/0000-0002-3412-7590

\title{
Le sens contextuel des « actes de langage stéréotypés »
}

\begin{abstract}
This contribution focuses on the contextual meaning of pragmatic phraseologisms, and especially what we call "stereotypical speech acts" in French, e.g. la belle affaire (big deal) or tu parles (you must be joking). We will first present the theoretical and methodological issues related to the notion of (linguistic or situational) context, and then analyze the relations between co(n)text and the meaning of "stereotypical speech acts".

Keywords: context, contextual meaning, pragmatic phraseologism, pragmateme, stereotypical speech act
\end{abstract}

Notre objectif est d'examiner le rôle du contexte et particulièrement du sens contextuel des phraséologismes, en prenant l'exemple des «actes de langage stéréotypés » (= ALS), catégorie de phraséologismes sur laquelle le GLFA (Groupe de lexicographie franco-allemande) travaille à l'ATILF (UMR CNRS/Université de Lorraine) à Nancy dans le cadre d'un projet de dictionnaire bilingue français/allemand de phraséologie. Nos exemples proviennent du 
corpus électronique constitué à cet effet: plus de 500 bi-textes littéraires en français et en allemand et leur traduction (60 millions de mots). Avant d'analyser les phraséologismes en texte ( $2^{\mathrm{e}}$ partie) et en contexte $\left(3^{\mathrm{e}}\right.$ partie), nous examinerons d'un œil critique la notion de contexte, particulièrement complexe $\left(1^{\mathrm{e}}\right.$ partie $)$.

\section{Le contexte : potentialités et dangers}

\subsection{Un contexte (in)définissable}

Le contexte est une notion à la fois essentielle, qui sert à résoudre des problèmes linguistiques et sémantiques, mais qui pose souvent problème elle-même, car elle a tant de dimensions qu'elle devient d'une grande complexité. On peut s'en rendre compte en examinant comment les linguistes sont souvent embarrassés en essayant de définir le contexte.

1.1.1 Le nombre de définitions existantes du contexte est extrêmement élevé. Sans vouloir toutes les citer, ni même les synthétiser, on se contentera de deux exemples particulièrement frappants. CrévenatWerner (1996) consacre un long article aux différentes définitions du contexte. Elle cherche pour cela du côté des lexicographes et des linguistes. Son travail de «débroussaillage» est fort utile, car il permet de dégager les points communs et les variantes existant entre les définitions du contexte en linguistique, mais elle est presque submergée par le nombre de définitions différentes.

Brézillon, président fondateur de l'AFC (Association Française pour le Contexte, créée en 2005), travaille sur le contexte dans le domaine de l'intelligence artificielle et par extension dans les sciences dites « dures ». Il a relevé (2006, p. 245) 165 définitions différentes du contexte sur le Web et recherche une définition universelle du contexte ou du moins des éléments récurrents pouvant servir à le définir. On lui souhaite bon courage.

1.1.2 Le contenu des définitions du contexte se signale par sa variété, mais en fait cette variété n'est pas si élevée. Si l'on examine quelques 
définitions du contexte, on s'aperçoit qu'elles sont très générales, voire vagues, comme si leurs auteurs ne voulaient pas prendre de risques en donnant une définition précise. Pour Kerbrat-Orecchioni (2009, p. 11), le contexte d'un item est «l'ensemble de ce qui accompagne, entoure, environne l'item en question ». C'est une définition assez générale et les trois verbes employés (en italiques, MK) sont quasi-synonymes. De même celle de Charaudeau \& Maingueneau (2002, p. 134): «Le contexte d'un élément $X$ quelconque, c'est en principe tout ce qui entoure cet élément». Le terme élément est plutôt vague et l'expression en principe modalise encore la définition. On pourrait multiplier les exemples. Kleiber (2009, p. 24) est déjà plus précis: il propose une «définition standard du contexte, à savoir celle d'un environnement qui exerce une influence sur les unités linguistiques ». En fait, les définitions ne deviennent plus précises que quand il s'agit:

- d'un domaine plus restreint du contexte (cotexte, contexte situationnel, contexte immédiat ou large etc.), voire d'une typologie du contexte

- des fonctions et influences du co(n)texte, évoquées ou détaillées par exemple dans Guimier (1997), Cusin-Berche (2003), Kerbrat-Orecchioni (2009), Kleiber (2009)

- du contexte en tant qu' «objet de confrontation entre théories » (Fillietaz, 2006, p. 71), par exemple dans les différentes analyses de Kerbrat-Orecchioni, Kleiber et Fillietaz (Fillietaz, 2006, p. 71).

Il semble donc qu'une définition précise n'est pas forcément un préalable à une analyse linguistique: il n'est peut-être pas nécessaire de passer par une définition pour travailler sur le contexte. On peut aussi procéder bottom up, donc trouver une définition seulement après un travail de fond sur des exemples précis de contexte.

\subsection{Un contexte dichotomique}

Dans un article de référence sur le contexte, Kleiber (1997, p. 13 et s.) propose 15 oppositions de base sur cette notion pour clarifier le problème. Nous allons revenir brièvement sur certaines d'entre elles, 
pour esquisser le cadre de notre analyse sur le contexte en phraséologie.

1.2.1 Caractère nécessaire ou non de l'analyse en contexte

Kleiber (1997; 2006; 2009) évalue les deux positions théoriques de base et leurs arguments. Peut-on se contenter d'analyser un élément, une phrase par exemple, sans contexte, c'est-à-dire en langue? Ou bien faut-il analyser l'énoncé en intégrant forcément le contexte ? Après avoir évalué les arguments de part et d'autre, il en arrive (Kleiber, 2009, p. 19 et Kerbrat-Orecchioni, 2012, p. 1) à réconcilier les différentes positions en remarquant que le contexte «n'est pas tout-puissant », mais que par ailleurs on ne peut parfois vraiment pas se passer de lui. Nous sommes pour notre part partisan d'une analyse qui intègre l'influence réciproque des éléments contextuels et de l'unité à analyser.

\subsubsection{Typologie structurée des contextes}

Les différentes théories qui se sont intéressées au contexte l'ont fait avec des méthodes parfois très différentes. On peut citer, sans détailler, les théories de l'énonciation, l'analyse conversationnelle, l'ethno-linguistique, la théorie de la contextualisation de Gumperz (Delhay, 1996) etc. ${ }^{1}$ Au-delà de leurs différences de fond souvent importantes, elles ont le mérite d'avoir approfondi les différents types de contexte ${ }^{2}$ et $d$ 'avoir abouti à une typologie dont voici quelques éléments importants :

- cotexte / contexte linguistique ou interne... vs contexte extralinguistique / situationnel (Schmoll, 1996, p. 237)

- contexte étroit / immédiat / micro-contexte vs contexte large / éloigné / macrocontexte

- contexte orienté, à droite ou à gauche par rapport à l'élément

\footnotetext{
${ }^{1}$ Elles sont à vrai dire souvent dans la «mouvance interactionniste » (Kerbrat, 2012, p. 1), pour qui le contexte est définitoire.

Kleiber (2009, p. 24) parle de «contexte comme cadre ou environnement structuré ».
} 
- contexte donné au départ ou construit au fur et à mesure du déroulement du discours et donc variable au fur et à mesure de l'évolution ${ }^{3}$. Le contexte peut donc être considéré comme statique ou bien dynamique car «le contexte est aussi soumis à un remodelage incessant au cours du déroulement de l'interaction » (Kerbrat-Orecchioni, 2009, p. 15).

Ces quelques dichotomies sont celles qui nous paraissent les plus utiles pour la suite de notre réflexion. Elles peuvent bien sûr se superposer, par ex. le contexte linguistique peut être par ailleurs étroit ou large. Il s'agit donc à la fois d'une typologie multifactorielle et à plusieurs niveaux, ce qui en fait toute sa richesse mais aussi sa complexité.

\subsection{Le contexte et ses effets}

On sait que «le contexte joue un rôle fondamental dans le fonctionnement des énoncés, en ce qui concerne les activités de production aussi bien que d'interprétation» (Charaudeau \& Maingueneau, 2002, p. 135). C'est bien sûr la dimension du contexte la plus en rapport avec le matériau linguistique et énonciatif et aussi la plus intéressante de par sa variété et la fréquence de ses occurrences. Les effets du contexte sur la production et l'interprétation du discours ont déjà été amplement étudiés. Il s'agit surtout de difficultés d'interprétation sémantique et donc de compréhension que le contexte - surtout situationnel - peut contribuer à résoudre. On trouvera une bonne vue d'ensemble et des exemples dignes d'intérêt dans Kleiber (2009, p. 20 et s.) et dans Kerbrat-Orecchioni (2009, p. 16 et s.), ainsi que des analyses de détail dans Charolles (1996) et Cusin-Berche (2003, pp. 20-26). Comme le montre Kleiber, ce sont soit des ambiguités syntaxiques ou lexicales, soit des énoncés « déviants » qui deviennent acceptables grâce au contexte, soit de l'«incomplétude interprétative » $(2009$, p. 23) c'est-à-dire les cas où un énoncé est imprécis, car le contexte situationnel nécessaire manque.

${ }^{3}$ A mettre en rapport avec le « contexte pour l'observateur » opposé au contexte pour les interlocuteurs » (Kleiber, 2009, p. 26 et s.). 
1.4 Un contexte fondamentalement problématique

Voici une dernière série de problèmes venant complexifier l'examen du contexte.

- Problèmes de délimitation: On se heurte souvent au fait qu'il est difficile de distinguer l'unité à analyser et son cotexte, car tous deux sont en fait de même nature, d'autant plus qu'au fur et à mesure du déroulement de l'analyse le cotexte devient souvent lui-même l'unité à analyser et réciproquement. La délimitation $\mathrm{du}$ contexte linguistique et du contexte situationnel est moins difficile, mais on peut se demander (Kerbrat-Orechioni, 2012, p.10) à quel stade de l'analyse il faut tenir compte du contexte situationnel, si ce dernier est effectivement indispensable et quel degré de généralité on doit attendre de son analyse.

- Problèmes de méthode : La plupart des travaux sur le contexte restent exclusivement théoriques et refusent d'adopter une démarche empirique ou sur corpus, pourtant souvent la seule à donner des résultats significatifs. Kleiber (2006, p. 18) constate: «il reste encore des 'emplois' à découvrir et à décrire » et «le 'descriptif', pourtant essentiel en matière de contexte, et la soumission à l'empirisme des faits n'ont pas tellement le vent en poupe ».

Kleiber relève aussi un important problème de méthode, lié au précédent, à savoir que les analyses partent en général de la valeur sémantique de l'unité analysée et cherchent à la retrouver dans les emplois contextuels. L'inverse, à savoir abstraire la valeur de l'unité à partir de ses emplois, est plus intéressant, mais aussi plus ardu. C'est pourtant le choix que nous ferons dans notre $3^{\mathrm{e}}$ partie.

- Problèmes de calcul: C'est un point essentiel, à la fois théorique et empirique. Il s'agit des moyens et procédures à définir et à appliquer pour le calcul et la modélisation du sens contextuel. Il s'agit de faire une analyse quantitative de l'influence du cotexte sur l'interprétation sémantique de l'énoncé et plus spécifiquement sur le sens des unités 
lexicales. Il y a peu de travaux dans ce domaine: on lira Desclés (1997) et Victorri (1997) dans l'ouvrage de Guimier (1997). Desclés met au point un modèle d'exploration contextuelle rigoureux qui comprend une procédure de recherche d'indices linguistiques pertinents dans le contexte. Victorri se consacre aux expressions polysémiques et cherche à modéliser les interactions de ces expressions avec leur contexte, en s'inspirant des réseaux connexionnistes. Une approche comparable est celle d'Anquetil (2009, p. 42) qui cherche à modéliser les paramètres contextuels pertinents pour interpréter des actes de langage indirects.

Ces méthodes de calcul du sens contextuel sont complexes, car il faut pouvoir (Guimier, 1997, p. 7 et s.) délimiter le cotexte par rapport au contexte situationnel, définir l'étendue du cotexte, déterminer quelles informations sémantiques sont apportées par le cotexte et lesquelles par l'unité à analyser, dégager les relations réciproques entre l'unité et les éléments du cotexte et élaborer une procédure qui intègre ces différents facteurs dans un modèle de calcul global. Même bâtie avec rigueur, «cette architecture présente aussi des limites : son application devra sans doute se limiter à des énoncés assez simples » (Victorri, 1997, p. 244).

Le problème est d'ailleurs que le calcul du sens contextuel se fait en général a posteriori. On cherche dans un énoncé déjà formé quels sont les éléments du cotexte susceptibles d'avoir une influence sur le sens de l'unité dont on analyse justement le contexte. Mais cette recherche a posteriori court le risque d'être en partie subjective: on trouve en fait ce que l'on veut bien trouver.

\section{Phraséologismes et texte}

Quels sont les relations entre phraséologismes et texte? C'est à vrai dire une thématique relativement peu étudiée, même dans les publications récentes. En effet, la recherche en phraséologie s'est surtout consacrée à l'inventaire des unités phraséologiques, à leur définition et leur délimitation, aux problèmes de classification et au «noyau dur » des phraséologismes, à savoir les idiomes. Elle ne s'est 
intéressée que récemment aux occurrences des phraséologismes (les tokens et non les types) et à l'intégration des phraséologismes dans les textes, cela grâce aux progrès de la linguistique de corpus et des méthodes d'exploration automatisée. Or, selon Edmonds (2016, p. 107), les phraséologismes « constituent une partie importante de notre activité langagière, avec des estimations qui vont de $25 \%$ (...) à 80 $\% »$. Leur emploi dans les textes est donc une donnée essentielle.

Les relations entre phraséologismes et textes ont été davantage étudiées chez les phraséologues de langue allemande. C'est donc de ce côté que nous chercherons des éléments de réponse, entre autres chez Burger (2010) et Sabban (2004) qui en synthétisent les résultats (voir aussi Mellado-Blanco, 2008, et une orientation bibliographique dans Burger (2010, p. 177). En voici les 3 axes principaux.

\subsection{Phraséologismes et type de texte}

Il y a beaucoup d'études de détail sur les phraséologismes employés dans différents types de texte, définis de façon plus ou moins précise. On verra Burger et al. (2007, chap. VII). Une étude méthodique exemplaire est aussi celle de Burger (2010, p. 168 et s.) qui résume les études sur les phraséologismes dans les textes de spécialité, la littérature de jeunesse et les émissions de télévision ${ }^{4}$.

Sabban $(2004$, p. 242 et s.) examine le problème de façon critique et se demande s'il y a une corrélation entre type de phraséologisme et type de texte, au niveau quantitatif et qualitatif. Elle constate qu'il n'y a pas de corrélation avérée entre type de phraséologisme et type de texte, seulement des tendances et des régularités. Par exemple, les formules de routine sont rares dans les publicités écrites, en revanche les phraséologismes modifiés y sont très fréquents.

\subsection{Phraséologismes et constitution du texte}

C'est un domaine mieux exploré et qui a abouti à des résultats intéressants. Les phraséologismes n'ont pas toujours été considérés comme dignes d'intérêt pour ce qui est de leur place et leur fonction

\footnotetext{
${ }^{4}$ Voir l'ouvrage collectif de Lenk \& Stein (2011) et aussi Sabban (2004).
} 
dans le texte (Sabban, 2004, p. 238). En effet, pour appréhender toutes leurs dimensions textuelles, il est nécessaire de les examiner sous leur forme «normale » (en tant qu'expression polylexicale), ou en tant que variante occasionnelle ayant parfois subi des modifications, ou sous l'angle de leur intégration textuelle et en particulier leur rôle dans la constitution du texte.

Le concept de base utilisé dans les publications allemandes à ce sujet est celui de textbildende Potenzen («potentialités de constitution du texte $»$ ), issu de la phraséologie russe, repris entre autres par Dobrovolskij (1980) et qui a connu plusieurs avatars. Selon ce concept, les phraséologismes ont des emplois qui contribuent de façon notable à la constitution du texte où ils se trouvent, ceci en relation avec sa fonction communicative (Sabban, 2004, p. 238; 242 et s.). Sabban fait notablement progresser cette notion en détaillant les emplois textuels des phraséologismes: soit un emploi «non marqué », comme simple «brique» (Baustein) du texte, soit un emploi "marqué » qui se décline de différentes façons: emploi dans des isotopies, des réseaux lexicaux, avec ou sans modification formelle. Sabban donne spécialement des exemples de phraséologismes dans des textes avec une «dimension affective» (textes humoristiques, ironiques etc.).

Burger (2010, p. 157) analyse également avec finesse les phraséologismes intervenant dans la constitution des textes. Il remarque que les phraséologismes très idiomatisés ne contribuent que peu à la constitution du texte, car ils ne participent guère aux procédés habituels de cohésion textuelle: pronominalisation, reprise anaphorique etc. Le locuteur peut certes reprendre le phraséologisme dans sa globalité, mais pas une de ses composantes, car cela entraînerait une (ré)apparition du sens littéral du phraséologisme. Par ex., après faire d'une pierre deux coups il n'est pas possible de reprendre pierre ou coups par une répétition ou un pronom. En revanche les «expressions à verbe support» n'y voient aucun inconvénient, en particulier pour leur composante nominale: le substantif n'est pas «bloqué » au niveau textuel, car le noyau sémantique (le substantif) et l'aspect processuel (le verbe) sont bien 
dissociés. Après mener des négociations, le substantif négociations peut être repris, pronominalisé, intégré dans un composé etc.

2.3. Modifications de phraséologismes et texte

L'emploi de phraséologismes subissant une modification formelle ou sémantique est un aspect très intéressant de leurs emplois textuels. C'est également un procédé de constitution du texte, mais qui n'existe que dans un contexte donné et n'est compréhensible que dans ce contexte.

Burger, distingue trois cas de figure selon que l'on a affaire à telle ou telle combinaison: modification de forme, modification de sens ou modification de forme et en même temps de sens. La modification de sens peut consister dans la présence simultanée du sens littéral et du sens figuré ou dans des éléments de sens ajoutés au sens figuré (2010, pp. 159-160). Il peut ainsi se créer dans le texte tout un réseau sémantique et formel partant du phraséologisme ou y aboutissant, ce qui contribue grandement à construire le texte. Un bon exemple de Burger montre que c'est souvent la substitution d'un élément du phraséologisme qui est à la base de la modification sémantique : $W C$ Ente verdient Ihr Vertrauen. ENTE gut, alles gut. Dans WC-Ente - un nettoyant WC à forme de canard (Ente) -, le substantif Ente se substitue à Ende ('fin') dans le proverbe Ende gut, alles gut ('Tout est bien qui finit bien'), qui devient un proverbe modifié où l'on suggère que ce nettoyant entraîne une fin heureuse, à savoir la propreté (2010, p. 161).

Mentionnons aussi que d'autres procédés et domaines où interviennent les phraséologismes peuvent avoir une dimension textuelle importante. C'est par exemple le défigement du phraséologisme, qui ne peut se faire en général que dans le cadre d'un contexte textuel donné. C'est aussi le cas en didactique des langues secondes où le nouveau phraséologisme à acquérir doit être présenté aux apprenants non pas de façon isolée, mais dans un texte, de préférence authentique. Son acquisition et son réemploi se font également en texte (Winzer-Kiontke, 2016, pp 310-311). Enfin, il en est de même en phraséographie bilingue où les dictionnaires actuels 
présentent le phraséologisme-vedette, sa traduction et les exemples avec leur contexte (Ruusila, 2015, p. 284 et s. et aussi le dictionnaire contextuel des ALS en cours d'élaboration à Nancy, cf. Kauffer 2018).

\section{Phraséologismes et contexte}

\subsection{Catégories et problèmes}

Pour analyser les relations entre phraséologismes et co(n)texte, il est particulièrement intéressant d'analyser les phraséologismes pragmatiques, à savoir une catégorie de phraséologismes dont les membres ont une fonction non pas seulement référentielle (comme en général les unités lexicales), mais essentiellement discursive et pragmatique et ont donc une grande sensibilité au contexte. La notion de «phraséologisme pragmatique »n'est pas récente ${ }^{5}$, mais est utilisée de plus en plus fréquemment et de nouveaux types de phraséologismes pragmatiques apparaissent régulièrement suite aux analyses sur la base de corpus textuels.

On distingue généralement deux grandes catégories de phraséologismes pragmatiques, même si les analyses divergent quelque peu selon les travaux.

\subsubsection{Les formules conversationnelles}

Le modèle récent le plus complet est celui de Stein (1995 et 2004), repris par une série de travaux ultérieurs (Hyvärinen, 2011; Ruusila, 2015; Winzer-Kiontke, 2016 etc.). Par manque de place, nous ne pourrons analyser ces formules, par ex. bref; comme prévu; en conclusion ; pas vrai ?; pour ce que j'en sais.

\footnotetext{
${ }^{5}$ Selon Ruusila (2015, p. 36), elle est employée pour la première fois par Burger dans Burger et al. (1982).
} 
3.1.2 Les pragmatèmes / formules de routine ${ }^{6}$

Ce sont soit des expressions, soit (assez fréquemment) des lexèmes uniques (bonjour ; feu ; fragile). Ils sont en général morphosyntaxiquement figés. Au niveau sémantique, la plupart ont un sens compositionnel, par exemple à table; au suivant ; bon rétablissement ; bon appétit. Mais ils peuvent aussi être de sens non- compositionnel : à vos souhaits ; garde à vous ; chaud devant ; à la tienne et ont souvent un caractère ritualisé.

Leur caractéristique principale est cependant d'être « pragmatiquement contraints », à savoir étroitement liés à la situation de leur emploi. Par exemple, à vos souhaits ne se dit que suite à l'éternuement de l'interlocuteur. Les pragmatèmes sont donc des «énoncés liés » comme le disaient déjà Fonagy (1997) et Schemann (1993, p. XXXVII).

Le terme de pragmatème est surtout employé dans les travaux de langue française, les collègues allemands (Coulmas, 1981; Schemann, 1993; Lüger, 2007; Burger, 2010; Hyvärinen, 2011; Ruusila, 2015; Winzer-Kiontke, 2016) parlent en général de formules de routine ${ }^{7}$.

\subsubsection{Problèmes}

C'est justement la «contrainte pragmatique », le critère définitoire de base des pragmatèmes, qui nous intéressera le plus dans notre réflexion sur le contexte. Ce lien étroit entre pragmatème et contexte situationnel a en fait été assez peu étudié mais il le mériterait, car il n'est en fait pas aussi évident qu'il en a l'air (cf. Kauffer, 2017). Pourquoi? S'il y a un lien étroit entre pragmatème et situation, il faut avoir à sa disposition une description précise de la situation et de ses paramètres. Or, ce n'est pas si facile (cf. l'essai de Blanco, 2014). On peut avoir recours aux actes de langage et à leurs caractéristiques pour cela, mais il n'est pas non plus toujours facile de traduire chaque pragmatème en termes d'actes de langage.

\footnotetext{
${ }^{6}$ Voir les analyses de Fléchon et al. (2012), Mel’čuk (2013), Blanco (2014), Kauffer (2017).

${ }^{7}$ Pour une typologie par fonction communicative, voir Ruusila (2015, p.74).
} 
D'autre part, le lien entre situation et pragmatème n'est pas absolu. En d'autres mots, il n'y a pas de lien biunivoque entre situation et pragmatème: un pragmatème n'est pas forcément lié à une seule situation. Par exemple, bonjour dans S'il vient lui aussi, bonjour l'ambiance! est employé par antiphrase : il exprime en fait au revoir! pour dire que la (bonne) ambiance va disparaître. De même, sincères condoléances dans $T u$ vas à cette réunion? Sincères condoléances! exprime le fait qu'un événement désagréable va arriver et qu'on exprime sa compassion à l'interlocuteur.

\subsection{ALS et contexte}

\subsubsection{Définition et délimitation}

Les travaux sur les pragmatèmes et formules conversationnelles négligent deux critères importants: le statut d'énoncé du phraséologisme et le contexte du phraséologisme, lié à son statut d'énoncé. Le contexte peut avoir des conséquences capitales sur le sens du phraséologisme. Approfondissons donc le problème à l'aide d'une catégorie particulière de phraséologismes: les actes de langage stéréotypés (ALS). Voici d'abord un bref rappel de leur trois critères définitoires. Le premier est leur statut d'énoncé. Un ALS peut toujours être un énoncé à part entière, par exemple tu parles! dans:

- T'as eu les jetons?

- Tu PARLES! Jamais eu une telle trouille de ma vie. (Queneau, 1967, p. 49)

Le deuxième est leur idiomaticité sémantique : Un ALS a un sens non-compositionnel, et donc habituellement un sens littéral et un sens phraséologique. Tu parles! dans l'exemple ci-dessus ne signifie pas seulement que le locuteur constate que son interlocuteur parle mais qu'il approuve fermement ce qui vient d'être dit. La différence entre sens littéral et phraséologique est très variable :

- soit les deux sens existent, avec une idiomaticité sémantique de l'ALS faible : c'est vite dit; tu parles; un point c'est tout

- soit les deux sens existent mais avec une idiomaticité forte : du balai; c'est le bouquet 
- soit seul le sens phraséologique existe : il faut voir comme ; tant qu'à faire.

On verra que ces différences sémantiques très variables sont liées au contexte d'emploi de l'ALS.

Le troisième critère est la fonction pragmatique. L'ALS est assimilable à un acte de langage ${ }^{8}$, avec une valeur illocutoire. Il ne sert pas seulement à dire quelque chose, mais aussi à faire, à agir, à changer la nature de la relation entre les interlocuteurs. Voici des exemples de fonctions pragmatiques, parmi beaucoup d'autres. L'ALS sert à :

- approuver ou confirmer : tu parles ; tu l'as dit ; et comment!

- rejeter : compte là-dessus ; tu peux toujours courir ; même pas en rêve

- menacer, avertir : essaie donc !; tu vas voir !; viens-y

- exprimer l'étonnement: tu vois ce que je vois ?; eh ben dis donc.

Un ALS peut non seulement être de nature performative, mais aussi exprimer une émotion, un sentiment (cf. les deux derniers exemples). Cette valeur affective peut se superposer à l'acte de langage proprement dit.

Il y a donc certains points communs et également des différences entre ALS et pragmatèmes ${ }^{9}$. Les principales différences concernent leurs emplois. Les ALS ont une «plasticité contextuelle » élevée que nous allons explorer, c'est-à-dire un sens variant beaucoup selon le contexte. Les pragmatèmes ont des sens plus restreints et en plus liés à une situation spécifique. On peut dire grossièrement que les pragmatèmes sont une sous-catégorie des ALS.

\subsubsection{Contextualité des ALS}

Quels sont les liens entre co(n)texte et ALS ?

\footnotetext{
${ }^{8} \mathrm{Au}$ sens d'Austin (1970) et Searle (1972).

${ }^{9}$ Dans Kauffer (à par. b) on trouvera une liste précise des points communs et des différences entre les deux catégories.
} 
3.2.2.1 Absence de « contrainte pragmatique »

Un ALS n'a pas de lien biunivoque avec une situation, contrairement au pragmatème. Il peut être utilisé dans des situations très différentes. Il n'y a donc pas de routinisation de l'ALS dans une situation donnée. Mais ceci n'exclut pas le fait qu'il y ait des liens entre ALS et contexte. Le pragmatème garde-à-vous ! s'emploie uniquement dans un contexte militaire et est provoqué par la présence d'un gradé. Mais l'ALS regardez-moi ça! exprime un commentaire critique utilisable avec des interlocuteurs très différents (pas seulement des militaires) et dans des situations très variées.

3.2.2.2 Relations anaphoriques avec le cotexte précédent

Il s'agit surtout de pronoms démonstratifs et personnels de reprise contenus dans l'ALS et reprenant un élément du cotexte précédent: ce, ça, le/la/les par ex. dans on dit ÇA; ÇA la fout mal ; je L'aurai ; tu me LE paieras.

En revanche, il est rare d'avoir une reprise ou une pronominalisation d'un élément de l'ALS vers le cotexte suivant, en particulier lorsqu'il s'agit d'un substantif car cela détruirait l'idiomatisation de l'ALS. Le substantif affaire de l'ALS la belle affaire n'est jamais repris ni pronominalisé dans les exemples de notre corpus.

\subsubsection{Relations déictiques}

Un ALS comprend assez souvent des indices déictiques qui permettent de préciser quelle est la situation et ses paramètres: temps, lieu, interlocuteurs. Ce sont surtout des pronoms personnels et des possessifs de la $1^{\mathrm{e}}$ et $2^{\mathrm{e}}$ personne, ainsi que des indications temporelles et spatiales :

- Pronoms: va $T E$ faire voir ; $T U M E$ la copieras, $T U$ parles ; $J E$ l'aurai ;

- Possessifs : MON œil; MA parole;

- Spatiaux : je vois ça d'ICI; je ne mange pas de ce pain-LÀ; allez voir $L \grave{A}-B A S$ si j' $Y$ suis. 
Ces indications déictiques, en particulier les pronoms personnels et les déterminants possessifs posent parfois problème. Elles ne réfèrent pas forcément à la situation dans laquelle l'ALS est énoncé: tu parles est utilisé même si le locuteur vouvoie son interlocuteur, mon ceil marque l'incrédulité du locuteur mais ne désigne nullement son œil. Ces éléments en principe déictiques sont figés, ils désignent un interlocuteur-type et non celui de la situation dans laquelle est employé l'ALS.

\subsubsection{Levée de la plasticité contextuelle}

Ce sont les nombreux cas où un même ALS peut avoir des sens très différents, voire contraires, selon le contexte. Il reste cependant un ALS dans tous les cas.

L'ALS tu parles (Métrich, 2012) peut servir à exprimer des actes de langage bien différents. Soit une contestation (ex. a), soit une approbation forte (ex. b).

(a) - Le sabre japonais ? Ça me semble pas très zen...

- Tu PARLES, C'EST GÉNIAL! Moi, quand j'ai le moral à zéro, je prends mon sabre et hop ! (Aubert, 2001, p. 71)

(b) $-\mathrm{T}$ 'as eu les jetons?

- TU PARLES! JAMAIS EU UNE TELLE TROUILLE DE MA VIE.

Le cotexte suivant sert à indiquer de quel sens de l'ALS il s'agit. Pour lever l'ambiguïté de l'ALS, le locuteur ajoute une précision après l'ALS: c'est génial montre qu'il conteste l'énoncé précédent (pas très zen). De même, jamais eu une telle trouille de ma vie marque qu'il l'approuve et renchérit même sur le fait d'avoir les jetons: il a eu en fait très peur.

Il n'y a donc pas pour les ALS de désémantisation ou d'érosion/blanchiment sémantique, c'est-à-dire de diminution de la complexité sémantique comme on l'indique souvent dans les études sur la pragmaticalisation (Kauffer à par. a). Au contraire, l'ALS présente une complexification sémantique qui pose souvent problème lorsqu'on part des nombreuses occurrences de l'ALS en contexte pour tenter de les subsumer. 


\subsubsection{Enrichissement contextuel}

Le contexte contribue à enrichir le sens de l'ALS, en général pour le renforcer. Il y a beaucoup de procédés qui vont dans ce sens.

- Renforcement par redoublement d'ALS

Dans l'exemple suivant, tu parles exprimant la contestation, le rejet, est renforcé par un autre ALS de sens proche, des clous, qui le suit immédiatement:

Qu'est-ce qu'elle avait pensé, que son grand-père était directeur des chemins de fer, peut-être? Ou au moins conducteur de locomotive? TU PARLES, DES CLOUS oui! Pas même un roulant, qu'il était. (Wolf, 1978, p. 41)

- Renforcement par éléments affectifs

On peut renforcer la dimension affective, émotive, de l'ALS en ajoutant des éléments qui marquent une émotion, un état psychologique se superposant à l'ALS et le renforçant. Dans l'exemple suivant, l'ALS et puis quoi encore! exprime le refus et ce refus se charge affectivement par toute une série d'éléments : un $2^{\mathrm{e}}$ ALS de sens proche (sans blague), une série d'énoncés exclamatifs (avec point d'exclamation), un juron (bordel), une interjection (allez), des expressions assez crues (faire chier, se faire enculer) et même un énoncé du locuteur, qui exprime son énervement (j'suis trop énervée). Ce renforcement par l'affectivité est très fréquent.

C'est un peu facile de s'abrutir à coup de tranquillisants ! Réapprenez à FAIRE CHIER, BORDEL ! On va tout de même pas SE FAIRE ENCULER la vie, et le soir faire de beaux rêves... ET PUIS QUOI ENCORE ! Non mais SANS Blague !... Allez, J'suis TROP ÉNERVÉE, j'vais m'en jeter un ! (Loeb, 1993, p. 84)

\subsubsection{6 (Dé)phraséologisation}

Le contexte est ici encore plus puissant, car il détermine si l'expression est un ALS ou non. Donc il peut contribuer à phraséologiser une expression ou au contraire à la déphraséologiser, lui refuser ce statut. C'est plutôt la déphraséologisation qui est courante, car la plupart des ALS n'existent justement qu'avec leur statut d'ALS : c'est mon petit doigt qui l'a dit ; vous m'en direz tant ; 
tant qu'à faire ; ça me fait une belle jambe. Le contexte a donc comme fonction d'indiquer que l'expression n'est pas un ALS. Par exemple l'ALS tu l'as dit peut être un ALS (ex a) servant à approuver un énoncé précédent:

(a) - Vivement la retraite, hein !

- TU L'AS DIT. Ça sent pas bon, toutes ces choses. (Izzo, 1996, p. 247)

Mais tu l'as dit peut ne pas être un ALS (ex b) et dans ce cas il sert à exprimer que le locuteur reprend ce qui a été dit auparavant:

(b) Aurélie. - (...) Pourquoi n'y a-t-il presque plus que des voleurs ?

Constance. - Parce que l'argent est le roi du monde.

Aurélie. - ENFIN. TU L'AS DIT. Nous y voILA. Parce que nous sommes dans le règne du veau d'or. (Giraudoux, 1945, p. 122)

Dans l'ex (a) il n'y a pas d'élément qui précise que tu l'as dit sert à approuver. C'est en quelque sorte le sens «non marqué » de tu l'as dit, qui est un ALS. Dans l'ex (b), à la fois nous y voilà et enfin indiquent qu'Aurélie attendait que Constance dise l'argent est le roi $d u$ monde. Ces éléments guident le lecteur vers une interprétation non phraséologique de tu l'as dit.

\section{Bilan}

Phraséologismes et co(n)texte ont donc des relations réciproques et très variées. Les phraséologismes servent à construire le texte et parfois à marquer le type de texte. Cela concerne un certain nombre de phraséologismes mais pas tous et surtout la direction du phraséologisme vers le contexte. D'autre part les phraséologismes fonctionnent en étroite symbiose avec le co(n)texte. Cela concerne surtout les phraséologismes pragmatiques et la direction du contexte vers le phraséologisme. Soit le contexte impose certains phraséologismes, à savoir les pragmatèmes, soit il a une influence très grande au niveau des emplois et du sens des ALS: relations anaphoriques et déictiques, levée de la plasticité contextuelle en cas de polysémie, enrichissement et renforcement contextuel en particulier au niveau affectif, (dé)phraséologisation et combinaison du sens littéral et phraséologique etc. 
Suite à notre réflexion, peut-être que les phraséologismes pragmatiques (pragmatèmes, formules de routine, formules conversationnelles et ALS) ne sont plus à considérer comme étant à la périphérie de la phraséologie traditionnelle. Ceci est dû au fait qu'ils ne remplissent pas toujours les trois critères traditionnels des phraséologismes: polylexicalité, idiomaticité sémantique et figement. En fait, ils seraient plutôt au centre, car ce sont les plus liés au contexte et à la situation et donc à leurs emplois. Benveniste (1966, p. 290) disait déjà: «le 'sens' d'une forme linguistique se définit par la totalité de ses emplois, par leur distribution et par les types de liaison qui en résultent »

\section{Bibliographie}

Anquetil, S. (2009). Données contextuelles et degré de conventionnalité dans les actes de langage indirects. In M. Sandré (Ed.), Analyse du discours et contexts (pp. 4152). Limoges: Lambert-Lucas.

Austin, J. L. (1970). Quand dire, c'est faire. Paris: Seuil.

Benveniste, E. (1966). Problèmes de liguistique générale, Vol. 1. Paris: Gallimard.

Blanco, X. (2014). Inventaire lexicographique d'une sous-classe de phrasèmes délaissée: les pragmatèmes. Cahiers de Lexicologie, 104, 133-153.

Brézillon, P. (2006). Vers d'autres contextes: entretien avec ... Pratiques, 129-130, 243-248.

Burger, H., Buhofer, A., \& Sialm, A. (Eds.) (1982). Handbuch der Phraseologie. Berlin: De Gruyter.

Burger, H., Dobrovol'skij, D., Kühn, P., \& Norrick, N. R. (2007). Phraseologie: ein internationales Handbuch zeitgenössischer Forschung, Vol. 1. Berlin: De Gruyter.

Charaudeau, P., \& Maingueneau, D. (2002). Dictionnaire d'analyse du discours. Paris: Seuil.

Charolles, M. (1996). Quand intervient le contexte dans la résolution des ambiguïtés? Scolia, 6, 163-184.

Coulmas, F. (1981). Routine im Gespräch. Zur pragmatischen Fundierung der Idiomatik. Wiesbaden: Athenaion.

Crévenat-Werner, D. (1996). Les contextes de contexte - La notion de contexte dans les dictionnaires. Scolia, 6, 13-38.

Cusin-Berche, F. (2003). Les mots et leur contexte. Paris: Presses Sorbonne Nouvelle. 
Delhay, C. (1996). Texte, contexte, contextualisation: a-t-on progressé? Scolia, 6, 6186.

Desclés, J.-P. (1997). Systèmes d'exploration contextuelle. In C. Guimier (Ed.), Cotexte et calcul du sens (pp. 215-232). Caen: PU de Caen.

Dobrovol'skij, D. (1980). Zur Dialektik des Begriffs der textbildenden Potenzen von Phraseologismen. Zeitschrift für Phonetik, Sprachwissenschaft und Kommunikationsforschung, 33(6), 690-700.

Edmonds, A. (2016). Le rôle du contexte dans le traitement des expressions conventionnelles. Cahiers de lexicologie, 108, 107-123.

Fillietaz, L. (2006). La place du contexte dans une approche praxéologique. Le cas de l'argumentation dans les interactions scolaires. Pratiques, 129-130, 71-88.

Fléchon, G., Frassi, P., \& Polguère, A. (2012). Les pragmatèmes ont-ils un charme indéfinissable? In P. Ligas \& P. Frassi (Eds.), Lexiques. Identités, Cultures (pp. 81-104). Vérone: QuiEdit.

Fónagy, I. (1997). Figement et changements sémantiques. In M. Martins-Baltar (Ed.), La locution entre langue et usages (pp.131-164). Paris: ENS Editions.

Guimier, C. (1997). Avant-propos. In C. Guimier (Ed.), Co-texte et calcul du sens (pp. 7-10). Caen: PU de Caen.

Hyvärinen, I., \& Liimatainen, A. (Eds.) (2011). Beiträge zur pragmatischen Phraseologie (= Finnische Beiträge zur Germanistik 25). Frankfurt/Main: Peter Lang.

Kauffer, M. (à par. a). De la pragmaticalisation en phraséologie. Actes du IVe congrès Phrasis Bucarest 2017, 12 p.

Kauffer, M. (à par. b). La pragmatique est-elle soluble dans la phraséologie ? Actes du Colloque "La Phraséologie Française: Débats théoriques et dimensions appliquées, 21- 22 septembre 2017 ». Arras: Université d'Artois, 10 p.

Kauffer, M. (2017). De la notion de pragmatème en phraséologie. In C. De Giovanni (Ed.), Fraseologia e paremiologia: passato, presente, future (pp.70-81). Milan: Franco Angeli.

Kauffer, M. (2018): Qu'est-ce qu'un ALS? Verbum XL, 1, 35-50.

Kerbrat-Orecchioni, C. (2009). Le traitement du contexte en analyse du discours en interaction. In M. Sandré (Ed.), Analyse du discours et contexts (pp. 9-16). Limoges: Lambert-Lucas.

Kerbrat-Orecchioni, C. (2012). Le contexte revisité. Corela, 1-24. Retrieved September 10, 2018, from http://corela.revues.org/2627.

Kleiber, G. (1997). Quand le contexte va, tout va et ... inversement. In C. Guimier (Ed.), Co-texte et calcul du sens (pp. 11-29). Caen: PU de Caen.

Kleiber, G. (2006). Introduction au(x) contexte(s). Pratiques, 129-130, 17-19. 
Kleiber, G. (2009). D'un contexte à l'autre: aspects et dimensions du contexte. L'information grammaticale, 123, 7-32.

Lenk, H., \& Stein, S. (Eds.). (2011). Phraseologismen in Textsorten (= Germanistische Linguistik, 211-212).

Mel'čuk, I. (2013). Tout ce que nous voulions savoir sur les phrasèmes, mais... Cahiers de lexicologie, 102, 129-149.

Mellado-Blanco, C. (Ed.). (2008). Beiträge zur Praseologie aus textueller Sicht. Hamburg: Verlag Dr. Kovac.

Métrich, R. (2012). Petit dictionnaire permanent des 'actes de langages stéréotypés' (ALS) - Microstructure de 'tu parles!'. Nouveaux Cahiers d'allemand, 1, 3-17.

Ruusila, A. (2015). Pragmatische Phraseologismen und ihre lexikografische Darstellung. Frankfurt am Main: Peter Lang.

Schemann, H. (1993). Deutsche Idiomatik - Die deutschen Redewendungen im Kontext. Stuttgart/Dresden: Klett Verlag.

Schmoll, P. (1996). Production et interprétation du sens: la notion de contexte est-elle opératoire? Scolia, 6, 235-255.

Searle, J. R. (1972). Les actes de langage - Essai de philosophie du langage. Paris: Hermann.

Stein, S. (1995). Formelhafte Sprache. Untersuchungen zu ihren pragmatischen und kognitiven Funktionen im gegenwärtigen Deutsch. Frankfurt am Main: Peter Lang.

Stein, S. (2004). Formelhaftigkeit und Routinen in mündlicher Kommunikation. In K. Steyer (Ed.), Wortverbindungen - mehr und weniger fest (pp. 262-288). Berlin: De Gruyter.

Victorri, B. (1997). Modéliser les interactions entre une expression polysémique et son co-texte. In C. Guimier (Ed.), Co-texte et calcul du sens (pp. 233-245). Caen: PU de Caen.

Winzer-Kiontke, B. (2016). Routineformeln als Lehr-/Lerngegenstand. München: Iudicium

\section{Sources}

Aubert, B. (2001). Descente d'organes. Paris: Seuil.

Giraudoux, J. (1945). La folle de Chaillot. Paris: Grasset.

Izzo, J. C (1996). Chourmo. Paris: Gallimard.

Loeb, C. (1993). Bon chic. Paris: Seuil.

Queneau, R. (1967). Zazie dans le métro. Paris: Gallimard.

Wolf, C. (1978). Kindheitsmuster. Darmstadt: Luchterhand, 1978. Traduction par G. Riccardi (1987): Trame d'enfance. Aix-en-Provence: Alinéa. 